\section{Medical Education During the COVID-19 Pandemic: Experience From a Newly Established Medical School}

We read with interest the recent article in the journal addressing medical education issues amid the COVID-19 pandemic [1]. We appreciate the authors for addressing the area of medical education during the COVID-19 pandemic. We would like to report our experience with undergraduate education at a newly established medical school, during the lockdown ensuing from this pandemic.

After the detection of the first COVID-19 case in Turkey on March 11, 2020, all universities were closed. Our institution had opened a year ago, and we have 48 undergraduate students. Our first year integrated curriculum was based on face-to-face interaction and laboratory sessions. Though, the past epidemics/ pandemics were one of the topics in the group discussions [2], yet we discovered that we ourselves were unprepared for providing medical education in this situation.

This disruption of education forced us to make a rapid transition to online teaching systems [3]. We discussed with the faculty members about adapting our program to distance education; and trained them for internet-based distance learning. After informing the students, we started providing our theoretical lectures online, as per a fixed schedule. Initially, the students' participation in the lectures was low. We tried to reach the non-participants to learn about their problems, and made an effort to keep them in the training system, which lead to increasing participation by students.
Before conducting the phase-end summative examination, we provided mock tests for the students, so as to get their feedback. In the end, we completed the program but had to postpone some laboratory sessions until the beginning of Phase 2.

We learned an important lesson that maintaining online programs successfully in medical education would need to be done through close communication with students. Moreover, despite the ever-present possibility of a pandemic arising at any time, medical schools were not ready for continuing their teaching. While face-to-face learning is the cornerstone of medical education, some distance-based educational activities might be incorporated as a routine in medical education, so as to ensure trained faculty and well-aware students.

Acknowledgement: Ass Prof Dr Ipek Gonullu for assisting in manuscript preparation.

Published online: July 15, 2020; PII: S097475591600210

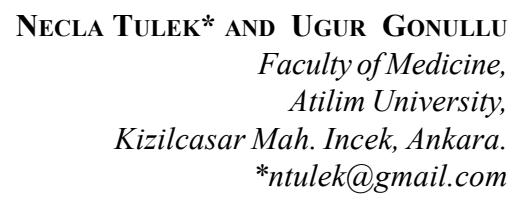

\section{REFERENCES}

1. Sahi PK, Mishra D, Singh T. Medical education amid the COVID-19 pandemic. Indian Pediatr. 2020 May 14;S097475591600181 [E-Pub Ahead of Print].

2. Aslan D, Sayek I. We need to rethink on the medical education for pandemic preparedness: Lessons learnt from COVID-19. Balkan Med J. 2020;37:178-79.

3. Torda AJ, Velan G, Perkovic V. The impact of COVID-19 pandemic on medical education. Med J Aust. 2020. Available from: https://www.mja.com.au/journal/2020/ impact-covid-19-pandemic-medicaleducation. Accessed June 01, 2020.

\section{Pediatric Coronavirus Disease-19 (COVID-19): Meta-analyzing Literature Versus Natural History}

We read with interest the recent systematic review on clinical features and outcome of severe acute respiratory syndrome coronavirus 2 (SARS-CoV-2) infection in children [1]. However, a major deficiency in the strategy seems to be the omission of Pediatric multisystem inflammatory syndrome (PMIS) [2]. Thus, the natural history of coronavirus disease 19 (COVID-19) in children seems innocuous. An early narrative review in the journal [3] observed that the mortality due to COVID-19 in children is rare, with majority being asymptomatic or having mild respiratory and gastrointestinal manifestations. The present systematic review [1] also substantiates that most children with COVID-19 were asymptomatic; amongst symptomatic children, only $0.7 \%$ required mechanical ventilation. The unique delayed cardiovascular manifestations in children have been omitted altogether from the suggested screening strategy for SARSCoV-2 infection.

In these circumstances, the elucidation of clinical features and outcome using a strategy of systematic review and metaanalysis is premature. The average time for the process of a systematic review is about 17 months [4]. On the other hand, a living systematic review methodology allows minimal loss to methodological rigor. It preserves and improves the currency, relevance, and usefulness of a systematic review. Technology is often applied for arduous data extraction processes. These facilitate efficient extraction of relevant data, and fast and maintained synthesis of evidence. The benefit for researchers and policy makers is also immense [4]. 


\section{CORRESPONDENCE}

The included studies in this review [1] were small and retrospective, with lot of heterogeneity and publication bias, the overall evidence generated is very low quality. Metaanalysis should be conducted with a group of homogeneous studies in terms of interventions involved and outcomes so as, to provide a meaningful summary [5].

Acknowledgement: Dr SK Vijesh for help in editing the manuscript.

P RAMESh Menon

CardioThoracic Sciences Center, All India Institute of Medical Sciences, New Delhi, India. rpmpgi@gmail.com

\section{REFERENCES}

1. Meena J, Yadav J, Saini L, Yadav A, Kumar J. Clinical features and outcome of SARS-CoV-2 infection in children: A systematic review and meta-analysis. Indian Pediatr. 2020; 57:820-6.

2. Kuttiatt VS, Abraham PR, Menon PR, Vaidya PC, Rahi M. COVID-19 children: Clinical and epidemiological implications. IJMR [E-pub ahead of print June 6, 2020].

3. Balasubramanian S, Rao NM, Goenka A, Roderick M, Ramanan AV. Coronavirus disease 2019 (COVID-19) in children - What we know so far and what we do not. Indian Pediatr. 2020;57:435-42.

4. Schmidt L, Olorisade BK, McGuinness LA, et al. Data extraction methods for systematic review (semi) automation: A living review protocol [version 1; peer review: Awaiting peer review]. F1000 Research. 2020;9:210.

5. Haidich AB. Meta-analysis in medical research. Hippokratia. 2010;14:29-37.

\section{AUTHORS'REPLY}

We appreciate the interest of the reader in our article [1]. The search of literature was performed till May 10, 2020, till when there were no published studies with ten or more patients describing the pediatric multisystem inflammatory syndrome (PMIS). It might be possible that there were a few case reports, but as mentioned in the methods, we did not include case reports and case series with less than ten cases. Therefore, this syndrome did not appear in our review.

We do not agree with the author's suggestion of including PMIS in the screening strategy for COVID-19 in children. As of now, PMIS is a rare and poorly understood presentation of COVID-19 in children [2]. The preliminary case definition itself is too complex to assess in the screening area [3]. Therefore, it might not be feasible to use it for the screening of COVID-19.
Living systematic review (LSR) is an emerging approach in which the review is updated frequently (classically at monthly intervals) and usually published online-only. Thought LSR seems a reasonable approach in COVID-19, it is very time consuming, requires lots of funding, and a dedicated team with long-term commitment. Moreover, agreement on methods to manage the data synthesis in LSR is still lacking, and the frequent statistical analyses can lead to an inflated falsepositive rate. Moreover, such a review can be published online only, therefore requiring a major change in the existing publication norms [4]. Therefore, at present, rather than considering it as a replacement, LSR should be considered as supplementary to the conventional review.

Ideally, meta-analysis should not have significant heterogeneity and the confidence interval should be very narrow. However, both of these conditions are extremely difficult to meet in observational studies, that too in the early stages of a pandemic. We explored heterogeneity using subgroup and sensitivity analysis using standard methods, but did not find any significant difference in the pooled estimates of any of the clinical or laboratory parameters. For a clinician, the knowledge of the pooled estimates for various clinical and laboratory conditions is indispensable, and it did not involve any intervention, therefore the meta-analysis was warranted. The limitations pointed out were already mentioned in our review [1].

JOGENDER KUMAR Department of Pediatrics, PGIMER, Chandigarh, India. jogendrayadav@gmail.com

\section{REFERENCES}

1. Meena J, Yadav J, Saini L, Yadav A, Kumar J. Clinical features and outcome of SARS-CoV-2 infection in children: A systematic review and meta-analysis. Indian Pediatr. 2020; 57:820-6.

2. Acharyya BC, Acharyya S, Das D. Novel coronavirus, mimicking kawasaki disease in an infant [published online ahead of print, 2020 May 22]. Indian Pediatr. 2020;:S097475591600184.

3. World Health Organization. Multisystem inflammatory syndrome in children and adolescents temporally related to COVID-19. Available from: https://www.who.int/newsroom/commentaries/detail/multisystem-inflammatorysyndrome-in-children-and-adolescents-with-covid-19. Accessed July 09, 2020.

4. Living Systematic Reviews. Available from: https:// community.cochrane.org/review-production/productionresources/living-systematic-reviews. Accessed July 07, 2020 